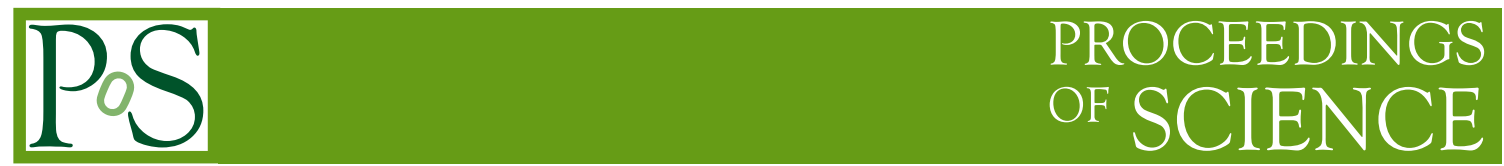

\title{
Recent progress on chiral symmetry breaking in
} QCD

\author{
Leonardo Giusti* \\ Dipartimento di Fisica, Università di Milano Bicocca \\ and INFN, Sezione di Milano Bicocca, \\ Piazza della Scienza 3, I-20126 Milano, Italy \\ E-mail: Leonardo.Giusti@mib.infn.it
}

I review recent progress achieved on the lattice in the quantitative comprehension of chiral symmetry breaking in QCD. Emphasis is given to the recent precise computations of the spectral density of the Dirac operator in the continuum limit, and of the topological susceptibility.

The 33rd International Symposium on Lattice Field Theory

14 -18 July 2015

Kobe International Conference Center, Kobe, Japan*

${ }^{*}$ Speaker. 


\section{Introduction}

There is overwhelming evidence from the lattice that the chiral symmetry group $\mathrm{SU}\left(N_{f}\right)_{L} \times$ $\mathrm{SU}\left(N_{f}\right)_{R}$ of the action of Quantum Chromodynamics (QCD) with a small number $N_{f}$ of massless flavors breaks spontaneously to $\mathrm{SU}\left(N_{f}\right)_{L+R}$. This progress became possible thanks to the steady increase of the computer power made available to our community, and to the impressive algorithmic and technical progress achieved over the last decade in the numerical simulation of lattice QCD with light dynamical fermions [1, 2, 3, 4, 5].

The Gell-Mann-Oakes-Renner (GMOR) relation was beautifully observed in the very first computations of this new generation of simulations already at finite lattice spacing $[6,7,8,9]$. Lattice results for ratios of low-lying eigenvalues of the Dirac operator in the $\varepsilon$ regime turned out to be in agreement with the parameter-free predictions of leading order (LO) Chiral perturbation theory (ChPT) and random matrix theory [10]. By now it is standard practice to assume the presence of spontaneous symmetry breaking in QCD, and fit phenomenologically interesting observables in the quark mass by applying the predictions of chiral perturbation theory (ChPT) [11, 12, 13]. After only 10 years from the first simulations with light quarks, we have many results in the $N_{f}=2$, $N_{f}=2+1$ and $N_{f}=2+1+1$ theories with light quarks down to the physical point, or lattice spacings as small as $0.05 \mathrm{fm}[14,15,16,17,18]$. There are many determinations of the QCD LO and next-to-leading order (NLO) low-energy constants (LECs) obtained by comparing the predictions of ChPT with lattice results, see Ref. [19] for a comprehensive review ${ }^{1}$.

Over the last decade we have also accumulated stronger and stronger evidence that the breaking due to the quantum anomaly of the $\mathrm{U}(1)_{L} \times \mathrm{U}(1)_{R}$ chiral group to $\mathrm{U}(1)_{L+R}$ is driven by the Witten-Veneziano mechanism [22, 23, 24]. After 10-15 years of exploratory computations with cooling techniques, well summarized in Ref. [25], a theoretically well defined definition of the cumulants of the topological charge was found [26, 27, 28, 29, 30, 31]. The value of the topological susceptibility obtained with the Neuberger definition of the charge [32] indeed supports the Witten-Veneziano explanation for the large experimental value of the $\eta^{\prime}$ mass. To properly verify the mechanism, however, precise computations at large $N_{c}$ with and without fermions are still required.

In the past two years or so, there have been further substantial conceptual, technical and numerical progress in our quantitative understanding of chiral symmetry breaking in QCD. It is the aim of this talk to review these advances with particular emphasis on the recent precise computations of the spectral density of the Dirac operator in the continuum limit, and of the cumulants of the topological charge distribution with and without fermions.

\section{Spectral density of the Dirac operator}

The spectral density of the Euclidean massless Dirac operator $D$ is defined as $[33,34,35]$

$$
\rho(\lambda, m)=\frac{1}{V} \sum_{k=1}^{\infty}\left\langle\delta\left(\lambda-\lambda_{k}\right)\right\rangle,
$$

${ }^{1}$ New (preliminary) determinations of some of the LECs of the SU(2) and SU(3) chiral effective theories of QCD with $N_{f}=2+1$ flavours which have reported at this conference $[20,21]$ quote errors significantly smaller than in Ref. [19]. 
where $i \lambda_{1}, i \lambda_{2}, \ldots$ are its (purely imaginary) eigenvalues, the bracket $\langle\ldots\rangle$ denotes the QCD expectation value, $V$ is the volume of the system, and $m$ is the quark mass. In QCD the density $\rho(\lambda, m)$ is a renormalizable quantity which is unambiguously defined once the free parameters in the action (coupling constant and quark masses) have been renormalized [36, 37]. The Banks-Casher relation [33]

$$
\lim _{\lambda \rightarrow 0} \lim _{m \rightarrow 0} \lim _{V \rightarrow \infty} \rho(\lambda, m)=\frac{\Sigma}{\pi}
$$

links the density at the origin to the chiral condensate

$$
\Sigma=-\frac{1}{2} \lim _{m \rightarrow 0} \lim _{V \rightarrow \infty}\langle\bar{\psi} \psi\rangle
$$

where $\psi$ is the quark doublet. It can be read in either directions: a non-zero spectral density at the origin implies that the symmetry is broken by a non-vanishing $\Sigma$ and vice versa. The mode number of the Dirac operator [36]

$$
v(\Lambda, m)=V \int_{-\Lambda}^{\Lambda} \mathrm{d} \lambda \rho(\lambda, m),
$$

turns out to be a renormalization-group invariant quantity as it stands. In presence of a non-zero chiral condensate the modes condense near the origin, and the mode number grows linearly with $\Lambda$. It can also be written as the average number of eigenmodes of the massive Hermitean operator $D^{\dagger} D+m^{2}$ with eigenvalues $\alpha \leq M^{2}=\Lambda^{2}+m^{2}$. Its (normalized) discrete derivative

$$
\tilde{\rho}\left(\Lambda_{1}, \Lambda_{2}, m\right)=\frac{\pi}{2 V} \frac{v\left(\Lambda_{2}\right)-v\left(\Lambda_{1}\right)}{\Lambda_{2}-\Lambda_{1}}
$$

carries the same information as $\rho(\lambda, m)$, but this effective spectral density is a more convenient quantity to consider in practice on the lattice.

\subsection{Mode number on the lattice}

On a discretized space-time and Wilson-type fermions, it is appropriate to define the mode number directly as the average number of eigenmodes of the squared massive Hermitean WilsonDirac operator $D_{m}^{\dagger} D_{m}$ with eigenvalues $\alpha \leq M^{2}$. In the continuum limit this definition converges to the universal one [36], with a rate proportional to $a^{2}$ if non-perturbative $\mathrm{O}(a)$-improved Wilson or Wilson twisted-mass fermions are implemented $[36,38]$. Since with those fermions chiral symmetry is explicitly broken at finite lattice spacing, the spectrum of the Wilson-Dirac operator near threshold $(\Lambda=0)$ is not protected from large discretization effects [37, 39, 40]. While this region may be of interest for studying the peculiar details of those fermions, it is easier to extract universal information about the continuum theory far away from it. This is one of the reasons for considering on the lattice the effective spectral density in Eq. (2.5).

\subsection{Mode number in ChPT}

When chiral symmetry is spontaneously broken, the mode number can be computed in the chiral effective theory. At the NLO it reads [41, 42, 36, 43, 39, 44]

$$
v^{\mathrm{nlo}}(\Lambda, m)=\frac{2 \Sigma \Lambda V}{\pi}\left\{1+\frac{m \Sigma}{(4 \pi)^{2} F^{4}}\left[3 \bar{l}_{6}+1-\ln (2)-3 \ln \left(\frac{\Sigma m}{F^{2} \bar{\mu}^{2}}\right)+f_{v}\left(\frac{\Lambda}{m}\right)\right]\right\}
$$


where

$$
f_{v}(x)=x\left[\arctan (x)-\frac{\pi}{2}\right]-\frac{1}{x} \arctan (x)-\ln (x)-\ln \left(1+x^{2}\right) .
$$

The constants $F$ and $\bar{l}_{6}$ are, respectively, the pion decay constant in the chiral limit and a SU(3|1) low-energy effective coupling renormalized at the scale $\bar{\mu}$. The formula in Eq. (2.6) has some interesting properties:

- for $x \rightarrow \infty$

$$
f_{v}(x) \stackrel{x \rightarrow \infty}{\longrightarrow}-3 \ln (x),
$$

and therefore at fixed $\Lambda$ the mode number has no chiral logarithms at the NLO when $m \rightarrow 0$;

- since in the continuum the operator $D_{m}^{\dagger} D_{m}$ has a threshold at $\alpha=m^{2}$, the mode number must satisfy

$$
\lim _{\Lambda \rightarrow 0} v^{\text {nlo }}(\Lambda, m)=0,
$$

a property which is inherited by the NLO ChPT formula;

- in the chiral limit $v^{\text {nlo }}(\Lambda, m) / \Lambda$ becomes independent on $\Lambda$. This is an accident of the $N_{f}=2$ ChPT theory at NLO [41];

- the $\Lambda$-dependence in the square brackets on the r.h.s. of Eq. (2.6) is parameter-free. Since $m \Sigma /\left(4 \pi F^{2}\right)^{2}>0$, the behaviour of the function $f_{v}(x)$ implies that $v^{\text {nlo }}(\Lambda, m) / \Lambda$ is a decreasing function of (small) $\Lambda$ at fixed (small) $m$.

At the NLO the effective spectral density $\tilde{\rho}^{\text {nlo }}$ inherits the same special properties of $v^{\text {nlo }}(\Lambda, m) / \Lambda$ : at fixed $\Lambda_{1}$ and $\Lambda_{2}$ it has no chiral logarithms when $m \rightarrow 0$, it is independent from $\Lambda_{1}$ and $\Lambda_{2}$ in the chiral limit, and at non-zero quark mass it is a decreasing parameter-free function of ${ }^{2}\left(\Lambda_{1}+\Lambda_{2}\right) / 2$. For light values of the quark masses, i.e. $10 \mathrm{MeV}$ or so, the variations are of the order of a few percent in the range of $\Lambda$ 's we are interested in. These special properties offer non-trivial tests that the values of $\Lambda$ and $m$ chosen in the simulations are in a regime where NLO ChPT can be applied.

\section{The spectral density in QCD Lite}

In the last two years the spectral density of QCD with two light flavours has been computed on a rich set of lattices with a statistical accuracy of a few percent [45, 46, 47]. The two groups opted for different gluonic and fermionic regularizations. The authors of Ref. [45] implemented the tree-level Symanzik improved gluon action and the Wilson twisted mass fermion action so to be able to use the gauge configurations generated by the ETM Collaboration. They span the parameter ranges ${ }^{3} a=0.054-0.085, m=16-47 \mathrm{MeV}$, and $M=50-120 \mathrm{MeV}$ (corresponding to approximatively $\Lambda=40-120 \mathrm{MeV}$ ). The authors of Refs. [46, 47] opted for the standard Wilson gluonic action and the non-perturbatively $\mathscr{O}(a)$-improved Wilson fermion action so to profit from the generation of the gauge configurations carried out by the CLS community ${ }^{4}$ and the Alpha

\footnotetext{
${ }^{2}$ It is very weakly dependent on $\left(\Lambda_{1}-\Lambda_{2}\right)$ for pairs of values $\Lambda_{1}$ and $\Lambda_{2}$ that we will consider.

${ }^{3}$ If not explicitly stated, the scheme- and scale-dependent quantities such as $\Sigma, m, \Lambda$ and $\rho$ are renormalized in the $\overline{\mathrm{MS}}$ scheme at $\mu=2 \mathrm{GeV}$.

${ }^{4}$ https://wiki-zeuthen.desy.de/CLS/CLS.
} 

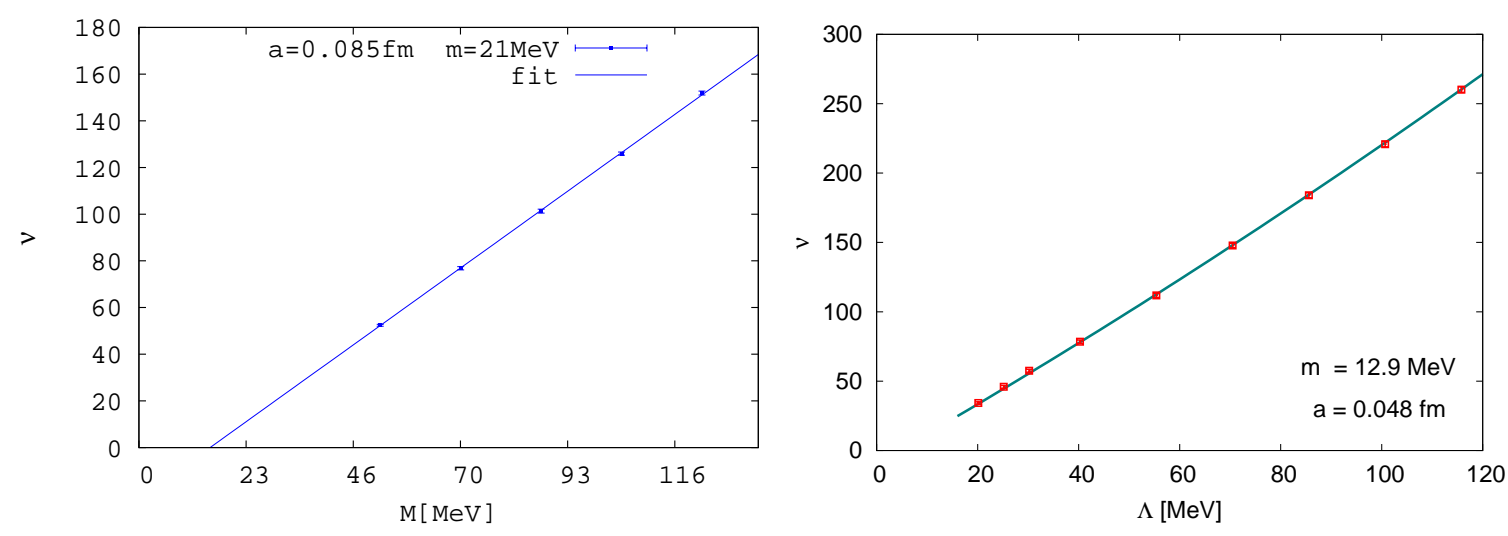

Figure 1: Left: the mode number $v$ as a function of $M$ for $a=0.085 \mathrm{fm}$ and $m=21 \mathrm{MeV}$ from Ref. [45]; the solid line is a linear fit to all 5 points. Right: the mode number as a function of $\Lambda$ for $a=0.048 \mathrm{fm}$ and $m=12.9 \mathrm{MeV}$ from Refs. [46, 47]; a quadratic fit of the data gives $v=-9.0(13)+2.07(7) \Lambda+0.0022(4) \Lambda^{2}$. Courtesy of Refs. [45, 46, 47].

collaboration $^{5}[48,15]$. The parameter ranges that they span are $a=0.048-0.075, m=6-37 \mathrm{MeV}$, and $\Lambda=20-500 \mathrm{MeV}$. In the left plot of Figure 1 the mode number is shown as a function of $M$ for a lattice with quark mass $m=21 \mathrm{MeV}$ and spacing $a=0.085 \mathrm{fm}$ from Ref. [45]. The right plot in the same figure shows $v$ as a function of $\Lambda$ for $m=12.9 \mathrm{MeV}$ and $a=0.048 \mathrm{fm}$ from Ref. [46, 47], a plot which makes manifest that the mode number is a nearly linear function of $\Lambda$ up to approximatively $100-150 \mathrm{MeV}$. The modes do condense near the origin as predicted by the Banks-Casher mechanism. At the percent precision, however, data show statistically significant deviations from the linear behavior already below $100 \mathrm{MeV}$. Just to guide the eye, a quadratic fit in $\Lambda$ is shown in the left plot of Figure 1 where is represented the set of data with lighter quark masses and which cover a wider range in $\Lambda$. The values of the coefficients, given in the caption, reveal that the bulk of $v$ is given by the linear term, while the constant and the quadratic term represent $\mathscr{O}(10 \%)$ corrections in the fitted range. This calls for a careful analysis of the systematics induced by cutoff effects, finite quark mass and finite $\Lambda$ values in order to reach a precise determination of the spectral density in the continuum and chiral limits at the origin.

\subsection{Continuum-limit extrapolation}

Since it is not affected by threshold effects, the effective spectral density $\tilde{\rho}$ in Eq. (2.5) is the primary observable the authors of Ref. [46, 47] focus on. The nearly linear behaviour of the mode number manifest itself in the (almost) flatness of $\tilde{\rho}$ in the same range. Because the action and the mode number are $\mathscr{O}(a)$-improved, the Symanzik effective theory analysis predicts that discretization errors start at $\mathscr{O}\left(a^{2}\right)$. In order to remove them, at every lattice spacing the authors of Ref. [46, 47] matched three quark mass values $(m=12.9,20.9,32.0 \mathrm{MeV})$ by interpolating $\tilde{\rho}$ linearly in $m$ for each value of $\Lambda$. Within the statistical errors all sets of data are compatible with a linear dependence in $a^{2}$, and thus each triplet of points are extrapolated linearly in $a^{2}$ to the continuum limit independently, e.g. see left plot in Figure 2. The results for $\tilde{\rho}$ at $m=12.9 \mathrm{MeV}$

\footnotetext{
${ }^{5}$ https://www-zeuthen.desy.de/alpha/
} 

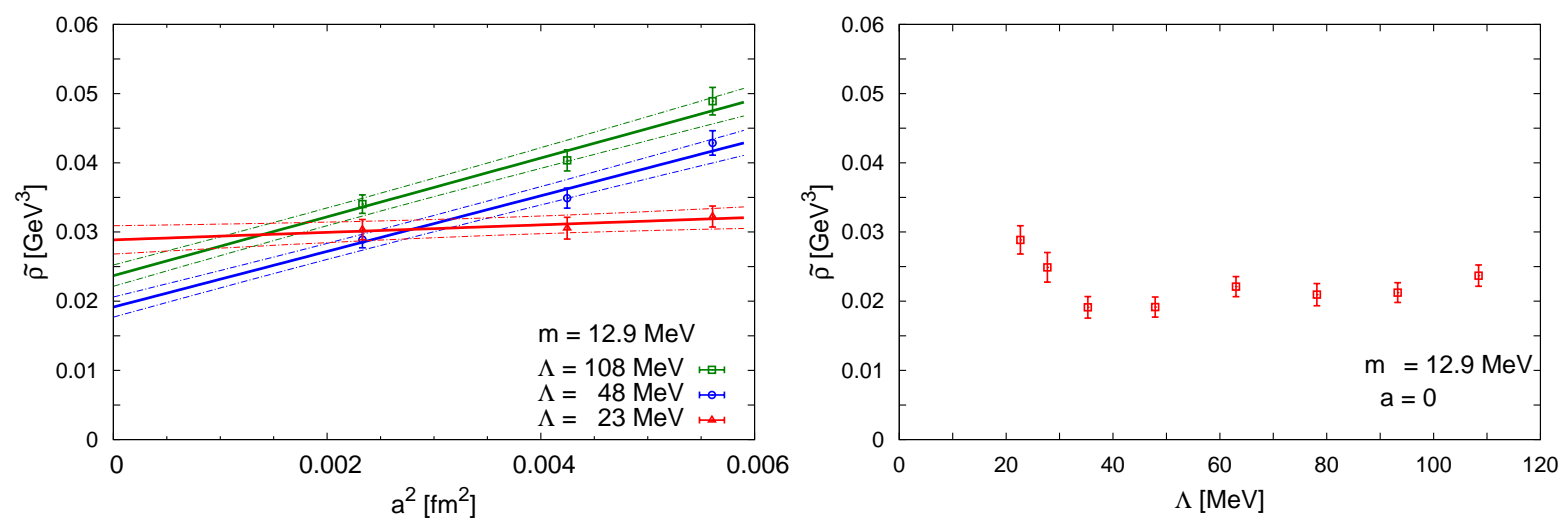

Figure 2: Left: continuum limit extrapolation of $\tilde{\rho}$ at the smallest reference quark mass $m=12.9 \mathrm{MeV}$ for three values of $\Lambda=\left(\Lambda_{1}+\Lambda_{2}\right) / 2$ [47]. Right: effective spectral density in the continuum limit as a function of $\Lambda=\left(\Lambda_{1}+\Lambda_{2}\right) / 2$ at the smallest reference quark mass $m=12.9 \mathrm{MeV}$ considered in Ref. [47]. Courtesy of Refs. [46, 47].

in the continuum limit are shown as a function of $\Lambda=\left(\Lambda_{1}+\Lambda_{2}\right) / 2$ in the right plot of the same Figure. A similar $\Lambda$-dependence is observed at the two other reference masses.

It is worth noting that no assumption on the presence of spontaneous symmetry breaking was needed so far. These results, however, point to the fact that the spectral density of the Dirac operator in two-flavour QCD is (almost) constant in $\Lambda$ near the origin at small quark masses. This is consistent with the expectation from the Banks-Casher relation in the presence of spontaneous symmetry breaking. As discussed in section 2.2, in this case the NLO ChPT indeed predicts an almost flat function in (small) $\Lambda$ at (small) finite quark masses which is parameter-free once the pion mass and decay constant are measured.

\subsection{Chiral limit}

The extrapolation to the chiral limit requires an assumption on how the effective spectral density behaves when $m \rightarrow 0$. The absence of chiral logarithms in Eq. (2.6), however, implies that $\tilde{\rho}^{\text {nlo }}$ is just a linear function of $m$ near the origin. In this limit, as discussed in section $2.2, \tilde{\rho}^{\text {nlo }}=\Sigma$ holds also at non-zero $\Lambda$ since all NLO corrections in Eq. (2.6) vanish. To check for this property, in Refs. $[46,47]$ the data have been extrapolated following Eq. (2.6) but leaving the leading term free to depend on $\Lambda$. The results of the fit are shown in the left plot of Figure 3. Within errors the $\Lambda$-dependence is clearly compatible with a constant up to $\approx 80 \mathrm{MeV}$. Moreover the difference between the values of $\tilde{\rho}$ in the chiral limit and those at $m=12.9 \mathrm{MeV}$ is of the order of the statistical error, i.e. the extrapolation is very mild. A fit to a constant of the data gives [46, 47]

$$
[\pi \rho]^{1 / 3}=261(6)(8) \mathrm{MeV},
$$

with the spacing being fixed in physical units by introducing a quenched strange quark and requiring that $F_{K}=109.6 \mathrm{MeV}$.

The authors of Ref. [45] analyzed their data by following the strategy adopted in the exploratory computation of Ref. [36]. The mode number at fixed lattice spacing and quark mass 

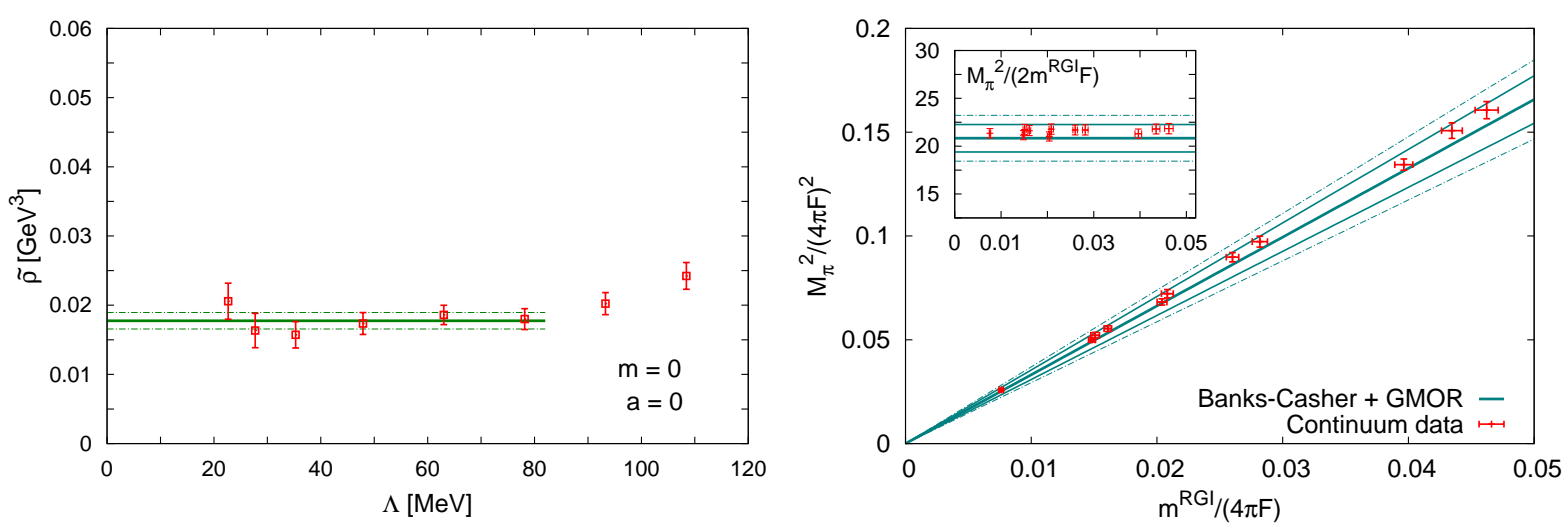

Figure 3: Left: effective spectral density $\tilde{\rho}$ in the continuum and chiral limits. Right: the continuum extrapolated pion mass squared versus the RGI quark mass, both normalized to $4 \pi F$. The central line is the GMOR contribution to the pion mass squared computed by taking the direct measure of the condensate from the spectral density. The upper and lower solid lines show the statistical error and the dotted-dashed ones the total error, the systematic being added in quadrature. Courtesy of Refs. [46, 47].

is fitted linearly in $M$ in the range $50 \leq M \leq 120 \mathrm{MeV}$. The slope is then linearly extrapolated in $m$ to the chiral limit, and the results are finally extrapolated to the continuum limit linearly in $a^{2}$, see left plot in Figure 4. The dependence of the slope on the fitting range is estimated and included in the systematic error. The result that they get is $r_{0}[\pi \rho]^{1 / 3}=0.689(16)(29)$. They prefer not to quote a value in $\mathrm{MeV}$ due to the large uncertainty in the determination of the lattice spacing in physical units from the ETM Collaboration [45]. However, if I use $r_{0} F_{K}=0.2794(44)$ from [15], I get $[\pi \rho]^{1 / 3}=270(6)(11)(4) \mathrm{MeV}$, an exercise that shows that their value is not inconsistent with the result in Eq. (3.1). More work by the ETM Collaboration is desirable to have a precise and reliable determination of the overall scale. For completeness in the right plot of Figure 4 the results for the analogous computation with $N_{f}=2+1+1$ is also shown [45].

\subsection{GMOR relation}

As in any numerical computation, the chiral limit inevitably requires an extrapolation of the results with a pre-defined functional form. The distinctive feature of spontaneous symmetry breaking, however, is that in the infinite volume limit the value of $\pi \rho$ at the origin has to agree with the one of $M_{\pi}^{2} F_{\pi}^{2} / 2 m$ in the chiral limit

$$
\lim _{m \rightarrow 0} \frac{M_{\pi}^{2} F_{\pi}^{2}}{2 m}=\pi \lim _{\lambda \rightarrow 0} \lim _{m \rightarrow 0} \rho(\lambda, m) .
$$

To make this comparison, the authors of Refs. [46, 47] complemented the computation of the mode number with those for the mass and the decay constant of the pion, $M_{\pi}$ and $F_{\pi}$, as well as of the quark mass $m$. The dimensionless ratio $M_{\pi}^{2} / 2 m F$, extrapolated to the continuum limit, is shown in the right plot of Figure 3 together with the GMOR contribution to it computed by taking the value of the spectral density in Eq. (3.1) (central line). The upper and lower solid lines show the statistical error and the dotted-dashed ones the total error, the systematic being added in quadrature. A different way to appreciate the excellent agreement is to compare the value of $[\pi \rho]^{1 / 3}$ reported in Eq. (3.1) with the one of the condensate extracted from the GMOR relation 

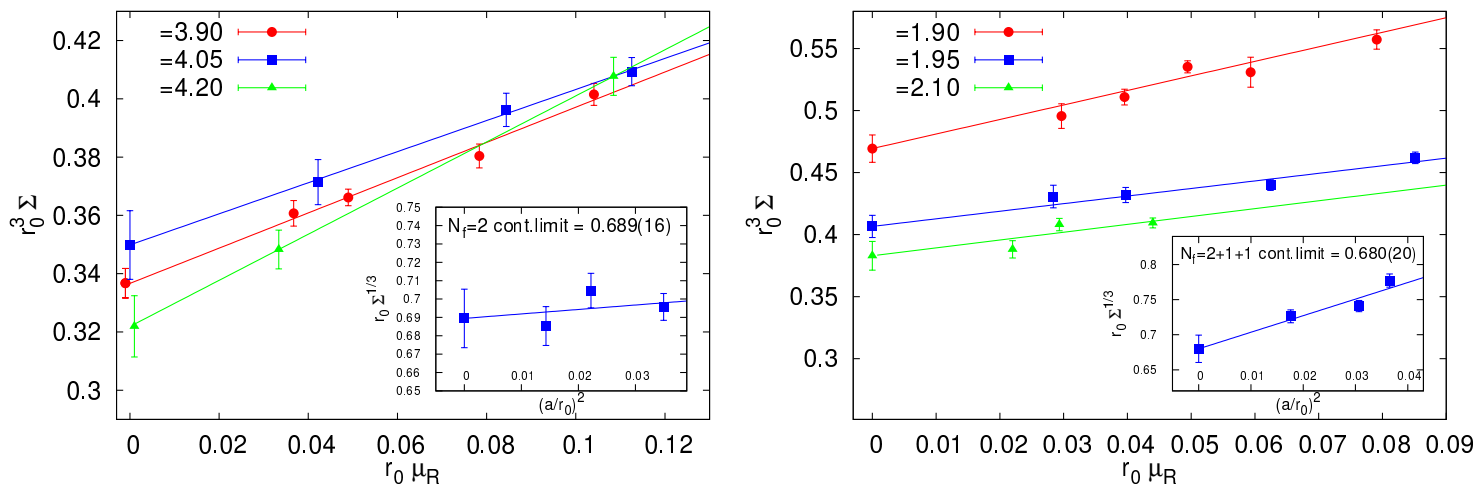

Figure 4: Chiral and continuum (inset) extrapolations of the spectral density for the $N_{f}=2$ (left) and $N_{f}=2+1+1$ (right) ensembles from Ref. [45]. Courtesy of Ref. [45].

which is $\left[\Sigma_{\mathrm{GMOR}}\right]^{1 / 3}=263(3)(4) \mathrm{MeV}$. For completeness the value obtained in Refs. [46, 47] for the decay constant in the chiral limit is $F=85.8(7)(20) \mathrm{MeV}$.

These results show that the spectral density of the Dirac operator in the continuum and chiral limits is non-zero at the origin. They provide a numerical proof of the fact that the low-modes of the Dirac operator do condense as dictated by the Banks-Casher mechanism in presence of a non-zero chiral condensate, and that the picture of spontaneously broken chiral symmetry in QCD is indeed correct.

A similar exercise can be done for the results in Ref. [45] and for those of the ETM collaboration in Ref. [49]. By taking $r_{0}=0.420(14)$ from [49] I obtain $[\pi \rho]^{1 / 3}=324(8)(14)(11)$, a value which has to be compared with the condensate extracted from the overall fit of the pion mass and the decay constant in the same reference, $\Sigma^{1 / 3}=269.9(65) \mathrm{MeV}$ [49]. The tension between the two values is again not inconsistent with the conclusions drawn above due to the large uncertainty (larger than the error quoted above) in the determination of $r_{0}$ in physical units from the ETM Collaboration, and also due to the discrepancy with respect to the value given before in units of $F_{K}$, see Refs. $[45,50]$ for a detailed discussion.

\subsection{Miscellaneous remarks}

From the analysis in Refs. [46, 47], the best results for the leading-order low-energy constants of QCD with two flavours are

$$
\left[\Sigma^{\overline{\mathrm{MS}}}(2 \mathrm{GeV})\right]^{1 / 3}=263(3)(4) \mathrm{MeV}, \quad F=85.8(7)(20) \mathrm{MeV} .
$$

By taking the value of the $\Lambda$-parameter from the Alpha collaboration [51, 15], and by taking into account the correlation with $F$, the results above correspond to the dimensionless ratios $[46,47]$

$$
\frac{\left[\Sigma^{\mathrm{RGI}}\right]^{1 / 3}}{F}=2.77(2)(4), \quad \frac{\Lambda^{\overline{\mathrm{MS}}}}{F}=3.6(2) .
$$

where the renormalization-group-invariant (RGI) condensate is defined with the convention of Refs. [52, 53]. For the sake of clarity, often (also in the previous sections) the values of dimensionful quantities in the $N_{f}=2$ theory are expressed in physical units. They are, however, affected by 
an intrinsic ambiguity due to the matching of the quantity chosen to fix the lattice spacing with its experimental value. The renormalization group-invariant dimensionless ratios quoted in Eq. (3.4), however, are parameter-free predictions of the $N_{f}=2$ theory. They belong to the family of unambiguous quantities that should be used for comparing results in the two flavour theory at variance with what is usually done in many reports, e.g. [19].

The quantities in Eq. (3.4) can be used to confront predictions from models, large $N_{c}$ approximation, etc. with lattice results. In this respect it is interesting to note that several years ago an analytic computation of the chiral condensate in QCD was carried out in the context of the planar equivalence between the large $N_{c}$ limits of the $\mathscr{N}=1$ super Yang-Mills theory and a variant of QCD with fermions in the antisymmetric representation $[54,55,56]$. In particular, an approximate expression for the quark condensate for $N_{c}=3 \mathrm{QCD}$ with quarks in the fundamental representation was inferred from an exact calculation of the gluino condensate in the $\mathscr{N}=1$ super Yang-Mills theory and further assumptions. The results that these authors quote, with the renormalization conventions adopted in Eq. (3.4), is

$$
\frac{\left[\Sigma^{\mathrm{RGI}}\right]^{1 / 3}}{\Lambda^{\overline{\mathrm{MS}}}}=1.43\left[\frac{N_{c}}{2 \pi^{2}} K_{\mathrm{F}}\left(1 / N_{c}, N_{f}\right)\right]^{1 / 3}
$$

where the numerical pre-factor 1.43 is just conventional and takes into account of the different conventions adopted in Refs. $[54,55,56]$ with respect to those in Eq. (3.4). The $K_{\mathrm{F}}$ function encodes the sub-dominant $1 / N_{c}$ corrections, which they conjecture to be relatively small. If I now compare the expression in Eq. (3.5) with the results in Eq. (3.4), I obtain $\left[K_{\mathrm{F}}(1 / 3,2)\right]^{1 / 3}=1.01(5)$ which, if taken at face value, is indeed compatible with having small sub-dominant corrections. Further lattice computations at various $N_{c}$ and $N_{f}$ are needed to test the conjecture, but the comparison above provides a good example on how the results in Eq. (3.4) can be used to test predictions from models and/or analytic approximations.

\section{The Witten-Veneziano relation and the gradient-flow}

The topological susceptibility in the $\mathrm{SU}\left(N_{c}\right)$ Yang-Mills theory can be formally defined in Euclidean space-time as

$$
\chi=\int d^{4} x\langle q(x) q(0)\rangle
$$

where the topological charge density $q(x)$ is

$$
q(x)=\frac{1}{64 \pi^{2}} \varepsilon_{\mu v \rho \sigma} F_{\mu \nu}^{a}(x) F_{\rho \sigma}^{a}(x) .
$$

In general the definition (4.2) of the topological charge density needs to be combined with an unambiguous renormalization condition. The cumulants of the charge, e.g. the susceptibility in Eq. (4.1), require also additional subtractions of short-distance singularities to make the correlators of the charge density integrable distributions. Besides its interest within the pure gauge theory, $\chi$ plays a crucial rôle in the QCD-based explanation of the large mass of the $\eta^{\prime}$ meson proposed by Witten and Veneziano (WV) $[23,24]$. Analogously to the GMOR relation, the WV mechanism predicts that at leading order in $1 / N_{\mathrm{c}}$ the contribution due to the anomaly to the mass of the 
pseudoscalar meson associated to the generator of $U(1)_{A}$ is given by [23, 24, 29, 57]

$$
\lim _{m \rightarrow 0} \lim _{N_{c} \rightarrow \infty} \frac{F_{\pi}^{2} m_{\eta^{\prime}}^{2}}{2 N_{\mathrm{f}}}=\lim _{N_{c} \rightarrow \infty} \chi
$$

For the Eq. (4.3) to be valid, the renormalization conditions for the charge and for the susceptibility need to be chosen so that in presence of fermions the anomalous chiral Ward identities are satisfied [29, 30, 31]. In this case the value of $\chi$ when $N_{c} \rightarrow \infty$ corresponds also to a low-energy constant in the simultaneous expansion in momenta and in $1 / N_{c}$ of the $\mathrm{U}(3)$ chiral effective theory $[58,59,60,61]$.

We know three families of definitions of the topological charge whose cumulants are ultraviolet finite and unambiguous: the one suggested by Ginsparg-Wilson (GW) fermions [26, 27, 28, $29,30,31$, the spectral projector formulas [36], and the naive definition at positive flow time [62]. When the topological charge is defined as suggested by GW, its bare lattice expression and those of the corresponding cumulants have finite and unambiguous continuum limits as they stand which satisfy the anomalous chiral Ward identities by construction. The continuum limit of this definition of $\chi$ is the right one to be inserted in Eq. (4.3). By combining a series of anomalous chiral Ward identities, the cumulants can be written as integrated correlation functions of scalar and pseudoscalalar density chains [30,31], and well chosen combinations of them correspond to the spectral projector definitions in Ref. [36]. Written in this form, a GW regularization is not required anymore to prove that no renormalization factor or subtractions of short-distance singularities are required. The spectral projector expressions thus provide a universal definition of the susceptibility and of the higher cumulants which, in the continuum limit, satisfy the anomalous chiral Ward Identities since in a chirally symmetric regularization they coincide with the GW ones.

Recently a third family of definitions of the topological charge was found [62], whose cumulants have a finite and unambiguous continuum limit [62,63]. It is a naive discretization of the charge evolved with the Yang-Mills gradient-flow. It is particularly appealing because its numerical evaluation is significantly cheaper than the others. This year there has been progress in this direction, and it has been shown that indeed the continuum limit of the cumulants of topological charge defined by the gradient-flow at positive flow time coincide with those of the universal definition. After more than 30 years, the long story of defining the topological charge on the lattice comes to a satisfactory end with a simple and elegant solution.

\subsection{Gradient-flow definition of the topological charge in the continuum}

Starting from the ordinary fundamental gauge field

$$
\left.B_{\mu}\right|_{t=0}=A_{\mu},
$$

the Yang-Mills gradient-flow evolves the gauge field as a function of the flow time $t \geq 0$ by solving the differential equation [62]

$$
\begin{aligned}
\partial_{t} B_{\mu} & =D_{v} G_{v \mu}+\alpha_{0} D_{\mu} \partial_{v} B_{v} \\
G_{\mu \nu} & =\partial_{\mu} B_{v}-\partial_{v} B_{\mu}-i\left[B_{\mu}, B_{v}\right], \quad D_{\mu}=\partial_{\mu}-i\left[B_{\mu}, \cdot\right],
\end{aligned}
$$


with $\alpha_{0}$ being the parameter which determines the gauge. Here we focus on the gradient-flow evolution of the topological charge density defined as

$$
q^{t}=\frac{1}{64 \pi^{2}} \varepsilon_{\mu v \rho \sigma} G_{\mu \nu}^{a} G_{\rho \sigma}^{a},
$$

and of the corresponding topological charge

$$
Q^{t}=\int d^{4} x q^{t}(x)
$$

When $q^{t}(x)$ is inserted in a correlation function at a physical distance with any finite (multi)local operator $O(y)$, it holds [64]

$$
\left\langle q^{t}(x) O(y)\right\rangle=\left\langle q^{t=0}(x) O(y)\right\rangle+\partial_{\rho} \int_{0}^{t} d t^{\prime}\left\langle w_{\rho}^{t^{\prime}}(x) O(y)\right\rangle \quad(x \neq y ; \rho=0, \ldots, 3),
$$

where $w_{\rho}^{t}$ is a dimension-5 gauge-invariant pseudovector field. The 1.h.s. of Eq. (4.9) is finite thanks to the fact that a gauge-invariant local composite field constructed with the gauge field evolved at positive flow time is finite [62,63]. Since there are no local composite fields of dimension $d<5$ with the symmetry properties of $w_{\rho}^{t}(x)$, the integrand on the r.h.s of Eq. (4.9) diverges at most logarithmically when $t^{\prime} \rightarrow 0$. This implies that the quantity

$$
\left\langle q^{t=0}(x) O(y)\right\rangle \equiv \lim _{t \rightarrow 0}\left\langle q^{t}(x) O(y)\right\rangle \quad(x \neq y),
$$

is finite, i.e. the limit on the r.h.s exists for any finite operator $O(y)$. The Eq. (4.10) can be taken as the definition of $q^{t=0}(x)$, i.e. the renormalized topological charge density operator at $t=0$. It is worth noting that Eq. (4.9) implies that the small- $t$ expansion of $q^{t}(x)$ is of the form

$$
\left\langle q^{t}(x) O(y)\right\rangle=\left\langle q^{t=0}(x) O(y)\right\rangle+\mathscr{O}(t) \quad(x \neq y),
$$

with no divergences when $t \rightarrow 0$.

In the following we are interested in supplementing the theory with extra degenerate valence quarks of mass $m$, and in considering the (integrated) correlator of a topological charge density with a chain made of scalar and pseudoscalar densities defined as [31]

$$
\left\langle q^{t=0}(0) P_{51}\left(z_{1}\right) S_{12}\left(z_{2}\right) S_{23}\left(z_{3}\right) S_{34}\left(z_{4}\right) S_{45}\left(z_{5}\right)\right\rangle,
$$

where $S_{i j}$ and $P_{i j}$ are the scalar and the pseudoscalar renormalized densities with flavor indices $i$ and $j$. Power counting and the operator product expansion predict that there are no non-integrable short-distance singularities when the coordinates of two or more densities in (4.12) tend to coincide among themselves or with 0 . When only one of the densities is close to $q^{t=0}(0)$, the operator product expansion predicts the leading singularity to be

$$
q^{t=0}(x) S_{i j}(0) \stackrel{x \rightarrow 0}{\longrightarrow} c(x) P_{i j}(0)+\ldots
$$

where $c(x)$ is a function which diverges as $|x|^{-4}$ when $|x| \rightarrow 0$, and the dots indicate sub-leading contributions. An analogous expression is valid for the pseudoscalar density. Being the leading short-distance singularity in the product of fields $q^{t=0}(x) S_{i j}(0)$, its Wilson coefficient $c(x)$ can be 
computed in perturbation theory. By using Eq. (4.9), to all orders in perturbation theory we can write

$$
\left\langle q^{t=0}(x) S_{i j}(0) O(y)\right\rangle=\left\langle q^{t}(x) S_{i j}(0) O(y)\right\rangle-\partial_{\rho} \int_{0}^{t} d t^{\prime}\left\langle w_{\rho}^{t^{\prime}}(x) S_{i j}(0) O(y)\right\rangle,
$$

where again $O(y)$ is any finite (multi)local operator inserted at a physical distance from 0 and $x$. When $t>0$, the first member on the r.h.s of Eq. (4.14) has no singularities when $|x| \rightarrow 0$. If present, the singularity has to come from the second term, and therefore $c(x)$ must be of the form

$$
c(x)=\partial_{\rho} u_{\rho}(x)
$$

which does not contribute to the integral (over all coordinates) of the correlation function (4.12).

\subsection{Ginsparg-Wilson definition of the charge density with the gradient-flow}

The definition of the topological charge density suggested by Ginsparg-Wilson fermions is [26, $28,27]$

$$
a^{4} q_{\mathrm{N}}^{t}(x)=-\frac{\bar{a}}{2} \operatorname{Tr}\left[\gamma_{5} D(x, x)\right],
$$

where we indicate it with a subscript $\mathrm{N}$ since, for concreteness, we take $D(x, y)$ to be the NeubergerDirac operator in which each link variable is replaced by the corresponding evolved one when $t>0$. Since there are no other operators of dimension $d \leq 4$ which are pseudoscalar and gauge-invariant, it holds that

$$
\lim _{a \rightarrow 0} Z_{q}\left\langle q_{\mathrm{N}}^{t}(0) q_{\mathrm{N}}^{t=0}(x)\right\rangle=\text { finite }
$$

where $Z_{q}$ is a renormalization constant which is at most logarithmically divergent, while $q_{\mathrm{N}}^{t}(0)$ is finite as it stands. This in turn implies that

$$
\lim _{a \rightarrow 0} Z_{q} a^{4} \sum_{x}\left\langle q_{\mathrm{N}}^{t}(0) q_{\mathrm{N}}^{t=0}(x)\right\rangle=\text { finite }
$$

since there are no short-distance singularities that contribute to the integrated correlation function because $q_{\mathrm{N}}^{t}(0)$ is evolved at positive flow-time. By supplementing the theory with extra degenerate valence quarks of mass $m$, and by replacing in Eq. (4.18) the topological charge at $t=0$ with its density-chain expression we obtain

$$
a^{4} \sum_{x}\left\langle q_{\mathrm{N}}^{t}(0) q_{\mathrm{N}}^{t=0}(x)\right\rangle=-m^{5} a^{20} \sum_{z_{1}, \ldots, z_{5}}\left\langle q_{\mathrm{N}}^{t}(0) P_{51}\left(z_{1}\right) S_{12}\left(z_{2}\right) S_{23}\left(z_{3}\right) S_{34}\left(z_{4}\right) S_{45}\left(z_{5}\right)\right\rangle .
$$

Written as in Eq. (4.19), power counting and the operator product expansion predict that there are no non-integrable short-distance singularities when the coordinates of two or more densities tend to coincide. The r.h.s of Eq. (4.19) is finite as it stands, and it converges to the continuum limit with a rate proportional to $a^{2}$. This in turn implies that the limits on the 1.h.s of Eqs. (4.17) and (4.18) are reached with the same rate if $Z_{q}$ is set to any fixed ( $g_{0}$-independent) value. Since in the classical continuum limit Neuberger's definition in Eq. (4.16) tends to the one in Eq. (4.7) [65, 66], we may set $Z_{q}=1$ in which case

$$
\lim _{a \rightarrow 0}\left\langle q_{\mathrm{N}}^{t=0}(x) O_{\mathrm{L}}(y)\right\rangle=\left\langle q^{t=0}(x) O(y)\right\rangle \quad(x \neq y),
$$

where $O_{\mathrm{L}}(y)$ is a discretization of the generic finite continuum operator $O(y)$. Once inserted in correlation functions at a physical distance from other (renormalized) fields, $q_{\mathrm{N}}^{t=0}(x)$ does not require any renormalization in the Yang-Mills theory. It is finite as it stands, and it satisfies the singlet Ward identities when fermions are included in the theory. So does $q^{t=0}(x)$. 


\subsection{Ginsparg-Wilson definition of the charge cumulants}

The Neuberger's definition of the topological charge is given by

$$
Q_{\mathrm{N}}^{t} \equiv a^{4} \sum_{x} q_{\mathrm{N}}^{t}(x)
$$

and its cumulants are defined as

$$
C_{\mathrm{N}, n}^{t}=a^{8 n-4} \sum_{x_{1}, \ldots, x_{2 n-1}}\left\langle q_{\mathrm{N}}^{t}\left(x_{1}\right) \ldots q_{\mathrm{N}}^{t}\left(x_{2 n-1}\right) q_{\mathrm{N}}^{t}(0)\right\rangle_{\mathrm{c}} .
$$

For $t=0$ the cumulants have an unambiguous universal continuum limit as they stand and, when fermions are included, they satisfy the proper singlet chiral Ward identities [29, 30, 31]. It is far from being obvious that $C_{\mathrm{N}, n}^{t=0}$ coincide with those defined at positive flow-time, since the two definitions may differ by additional finite contributions from short-distance singularities.

For the clarity of the presentation we start by focusing on the lowest cumulant, the topological susceptibility $C_{\mathrm{N}, 1}^{t}$. At $t=0$, by replacing one of the two $q_{\mathrm{N}}^{t=0}$ with its density-chain expression [31], we obtain

$$
a^{4} \sum_{x}\left\langle q_{\mathrm{N}}^{t=0}(0) q_{\mathrm{N}}^{t=0}(x)\right\rangle=-m^{5} a^{20} \sum_{z_{1}, \ldots, z_{5}}\left\langle q_{\mathrm{N}}^{t=0}(0) P_{51}\left(z_{1}\right) S_{12}\left(z_{2}\right) S_{23}\left(z_{3}\right) S_{34}\left(z_{4}\right) S_{45}\left(z_{5}\right)\right\rangle .
$$

When the susceptibility is written in this form, the discussion toward the end of section 4.1 and in particular Eq. (4.15) guarantee that there are no contributions from short-distance singularities. This result, together with the fact that $Z_{q}=1$, implies that

$$
\lim _{t \rightarrow 0} \lim _{a \rightarrow 0} a^{4} \sum_{x}\left\langle q_{\mathrm{N}}^{t}(x) q_{\mathrm{N}}^{t=0}(0)\right\rangle=\lim _{a \rightarrow 0} a^{4} \sum_{x}\left\langle q_{\mathrm{N}}^{t=0}(x) q_{\mathrm{N}}^{t=0}(0)\right\rangle .
$$

By replacing on the 1.h.s $q_{\mathrm{N}}^{t=0}(0)$ with the evolved one, no further short-distance singularities are introduced and we arrive to the final result

$$
\lim _{t \rightarrow 0} \lim _{a \rightarrow 0} a^{4} \sum_{x}\left\langle q_{\mathrm{N}}^{t}(x) q_{\mathrm{N}}^{t}(0)\right\rangle=\lim _{a \rightarrow 0} a^{4} \sum_{x}\left\langle q_{\mathrm{N}}^{t=0}(x) q_{\mathrm{N}}^{t=0}(0)\right\rangle .
$$

By replacing $2 n-1$ of the charges in the $n^{\text {th }}$ cumulant with their density-chain definitions, the very same line of argumentation can be applied. The Eq. (4.25), together with the independence up to harmless discretization effects of $C_{\mathrm{N}, n}^{t}$ from the flow-time for $t>0$ [62], implies that the continuum limit of $C_{\mathrm{N}, n}^{t}$ coincides with the one of $C_{\mathrm{N}, n}^{t=0}$. The cumulants of the topological charge distribution defined at $t>0$ thus satisfy the proper singlet chiral Ward identities when fermions are included. They are the proper quantities to be inserted in the Witten-Veneziano relations for the mass and scattering amplitudes of the $\eta^{\prime}$ meson in QCD.

\subsection{Universality at positive flow-time}

For $t>0$ different lattice definitions of the topological charge density tend to the same continuum limit if they share the same asymptotic behavior in the classical continuum limit $[62,63]$. We can therefore consider the naive definition of the topological charge density defined as

$$
q^{t}(x)=\frac{1}{64 \pi^{2}} \varepsilon_{\mu v \rho \sigma} G_{\mu \nu}^{a}(x) G_{\rho \sigma}^{a}(x),
$$


where the field strength tensor $G_{\mu \nu}^{a}(x)$ is defined as

$$
G_{\mu \nu}^{a}(x)=-\frac{i}{4 a^{2}} \operatorname{Tr}\left[\left(Q_{\mu \nu}(x)-Q_{\nu \mu}(x)\right) T^{a}\right],
$$

with

$$
\begin{aligned}
Q_{\mu v}(x)= & V_{\mu}(x) V_{v}(x+a \hat{\mu}) V_{\mu}^{\dagger}(x+a \hat{v}) V_{v}^{\dagger}(x)+ \\
& V_{v}(x) V_{\mu}^{\dagger}(x-a \hat{\mu}+a \hat{v}) V_{v}^{\dagger}(x-a \hat{\mu}) V_{\mu}(x-a \hat{\mu})+ \\
& V_{\mu}^{\dagger}(x-a \hat{\mu}) V_{v}^{\dagger}(x-a \hat{\mu}-a \hat{v}) V_{\mu}(x-a \hat{\mu}-a \hat{v}) V_{v}(x-a \hat{v})+ \\
& V_{v}^{\dagger}(x-a \hat{v}) V_{\mu}(x-a \hat{v}) V_{v}(x+a \hat{\mu}-a \hat{v}) V_{\mu}^{\dagger}(x) .
\end{aligned}
$$

In the Yang-Mills theory $q^{t=0}(x)$ requires a multiplicative renormalization constant when inserted in correlation functions at a physical distance from other operators [67]. The cumulants of the corresponding topological charge, defined analogously to Eq. (4.22), have additional ultraviolet power-divergent singularities, and they do not have a continuum limit.

The density $q^{t}(x)$ in Eq. (4.26) shares with $q_{\mathrm{N}}^{t}(x)$ the same asymptotic behavior in the classical continuum limit [65, 66]. Since for $t>0$ short-distance singularities cannot arise, $C_{\mathrm{N}, n}^{t}$ and $C_{n}^{t}$ tend to the same continuum limit. The results in the previous section then imply that the continuum limit of the naive definition of $C_{n}^{t}$, at positive flow-time, coincides with the universal definition which satisfies the chiral Ward identities when fermions are added [29, 30, 31]. It is interesting to note, however, that at fixed lattice spacing there can be quite some differences. For instance, the topological susceptibility defined at $t>0$ with the naive definition is not guaranteed to go to zero in the chiral limit at finite lattice spacing in presence of fermions [68].

\section{First two cumulants of the charge distribution in the SU(3) Yang-Mills theory}

Over the last year or so the topological susceptibility of the SU(3) Yang-Mills theory has been computed by several groups on a rich set of lattices with a statistical error of a few percent $[69,70,64,71]$. The results are shown in the left plot of Figure 5 together with the older results obtained with the Neuberger [32] and with the spectral projector [72] definitions of the charge. The best continuum limit values from the three families of definitions are ${ }^{6}$

$$
\begin{array}{ll}
r_{0}^{4} \chi=0.0544 \pm 0.0018 & \text { gradient-flow [64] } \\
r_{0}^{4} \chi=0.059 \pm 0.003 & \text { Neuberger definition [32] } \\
r_{0}^{4} \chi=0.049 \pm 0.006 & \text { modified spectral projector [70] }
\end{array}
$$

see left plot in Figure 5. They all agree within less than 2 standard deviations. By computing the topological susceptibility at different flow times, one can determine the ratios $\chi_{t} / \chi$ for which the statistical correlations among data reduce the error significantly. In Ref. [64] the continuum limit of this ratio was found to be compatible with 1 at the permille level at various values of $t$, another

\footnotetext{
${ }^{6}$ Unless explicitly indicated, the gradient flow-time at which the topological quantities are computed throughout this and the next section is at $t=t_{0}$.
} 

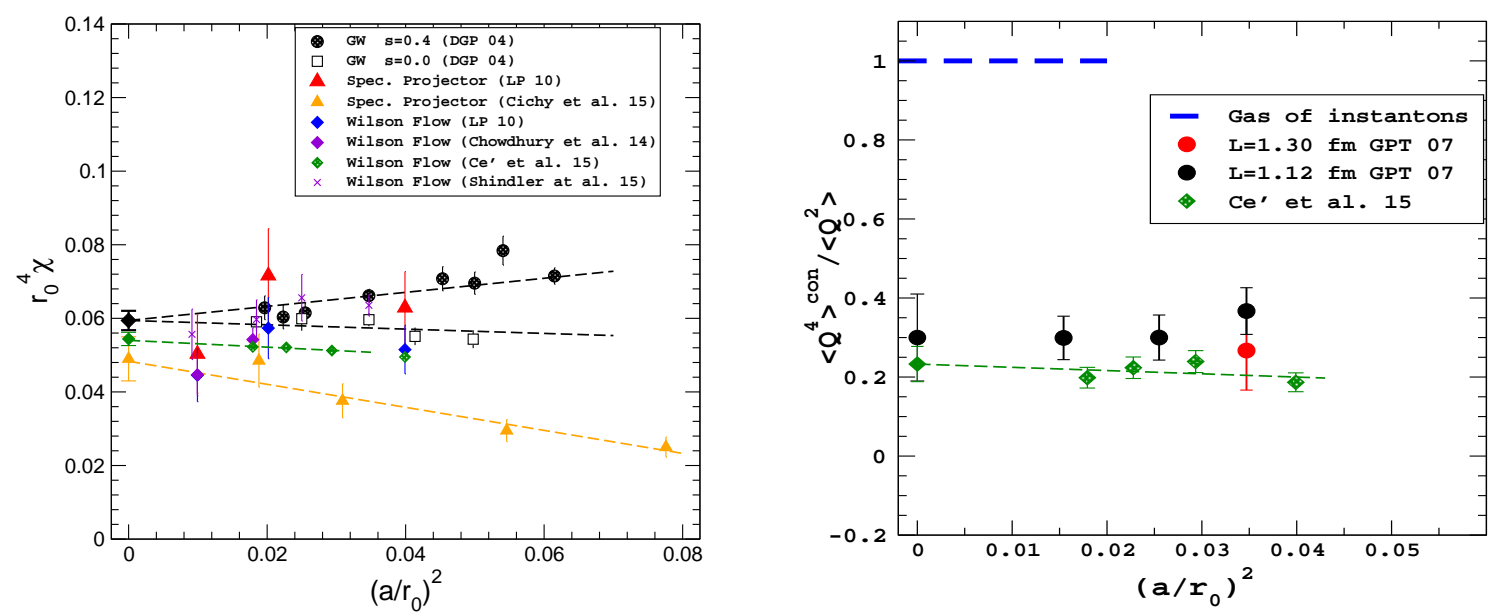

Figure 5: The topological susceptibility $\chi$ (left) and the ratio $R=\left\langle Q^{4}\right\rangle_{c} /\left\langle Q^{2}\right\rangle$ in the $\mathrm{SU}(3)$ Yang-Mills theory versus $\left(a / r_{0}\right)^{2}$.

universality test far from being trivial. At finite lattice spacing, in fact, discretization effects are clearly visible, and they depend on $t$. All these numerical results are consistent with the conceptual progress made over the last decade. Up to date there is no sign of non-universal behaviour of $\chi$ in the continuum limit, if the topological charge is properly defined on the lattice. We have moved from an unsolved problem in quantum field theory to precise universality tests!

The numerical computation of the charge defined by the gradient-flow is orders of magnitude cheaper than for the other two expressions. Since its cumulants at positive flow time coincide with those of the universal definition and discretization effects remain mild, the flow expression becomes the way to go for further (precise) studies of topological observables on the lattice. Given the statistical precision that can be easily reached, the all-time favorite Sommer reference scale $r_{0}$ [73] needs to be replaced by quantities which can be determined with a higher statistical (and systematic) precision, such as those derived from the gradient-flow [50]. By using $t_{0}$ as a reference scale [62], the authors of Ref. [64] obtain the most precise determination of the topological susceptibility in the SU(3) Yang-Mills theory to date

$$
t_{0}^{2} \chi=(6.67 \pm 0.07) \cdot 10^{-4}
$$

a value which is almost 3 times more precise than the one that can be obtained by using the present best determination of $r_{0}$.

In Ref. [64] the ratio of the second cumulant over the topological susceptibility, $R=\left\langle Q^{4}\right\rangle_{c} /\left\langle Q^{2}\right\rangle$, was also computed with the gradient-flow definition by keeping for the first time all systematics, especially finite volume effects, negligible with respect to the statistical errors. The results are shown in green on the right plot of Fig. 5. Their continuum limit extrapolation is

$$
R=0.233 \pm 0.045
$$

which is in agreement with a previous determination obtained with the GW definition [74], black and red points in the right plot of Fig. 5, albeit with an error 2.5 times smaller. The result in Eq. (5.3) is incompatible with the $\theta$-behaviour of the vacuum energy predicted by dilute instanton 
models, for which $R=1$ [75]. It suggests that the quantum fluctuations of the topological charge are of quantum non-perturbative nature in the ensemble of gauge configurations that dominate the path integral. The large $N_{c}$ expansion does not provide a sharp prediction for the value of $R$. Its small value, however, is compatible with being a quantity suppressed as $1 / N_{c}^{2}$ in the large $N_{c}$ limit.

\section{Topological susceptibility in QCD}

The computation of the topological susceptibility is expensive with respect to other quantities computed in (full) QCD. This is due to the long autocorrelation encountered in the simulations [76, 77, 78], and to the intrinsic fluctuations of this quantity. By expanding the charge distribution around the leading Gaussian behaviour, the Edgeworth expansion leads to the estimate of the relative error for the susceptibility given by $\Delta \chi / \chi=\sqrt{2 / N_{\text {conf }}}+\ldots$, where the dots indicate terms which are suppressed by powers of $1 / V$ [74]. For a precision of $\sim 5 \%, \mathscr{O}(1000)$ independent configurations are needed.

In the presence of spontaneous symmetry breaking with $N_{f}$ degenerate light flavours, the topological susceptibility toward the chiral limit goes as

$$
\chi_{\mathrm{QCD}}=\frac{\Sigma}{N_{f}} m+\mathscr{O}\left(m^{2}\right),
$$

which implies a significant suppression with respect to the Yang-Mills theory. Moreover being a pure gluonic operator, the slope of $\chi_{\mathrm{QCD}}$ is a measurement a posteriori of the number of flavours simulated once the chiral condensate is known. Vice versa, given the cost of its computation, the topological susceptibility is not a competitive quantity to determine $\Sigma$.

In the last couple of years there have been two computations of $\chi_{\mathrm{QCD}}$, both carried out on lattices with $\mathscr{O}(100)$ independent gauge configurations ${ }^{7}$. The statistical errors are therefore still quite large. The Alpha collaboration computed the susceptibility, defined from the gradient-flow charge density as in Eqs. (4.26), in the $N_{f}=2$ theory discretized with the standard Wilson gluonic action and the non-perturbatively $\mathscr{O}(a)$-improved Wilson fermion action. They used the gauge configurations generated by the CLS community and by themselves to obtain the results shown in the left plot of Figure 6 [68]. The ETM collaboration computed the susceptibility by implementing a variant of the spectral projection definition in the $N_{f}=2$ and $N_{f}=2+1+1$ theories. They have simulated the tree-level Symanzink $\left(N_{f}=2\right)$ and the Iwasaki gluon actions $\left(N_{f}=2+1+1\right)$, and the Wilson twisted mass fermion action. The results that they have obtained in the $N_{f}=2+1+1$ theory is shown in the right plot of Figure 6 as a function of the renormalized quark mass [80].

The suppression of $\chi_{\mathrm{QCD}}$ with respect to the Yang-Mills result is clearly seen in both sets of results, and it is manifest in the left plot where also the Yang-Mills value of the susceptibility is reported. In either cases there is no reason for $\chi_{\mathrm{QCD}}$ to vanish in the chiral limit at finite lattice spacing. The authors of Ref. [68] indeed extrapolate the data with the functional form suggested by LO Wilson ChPT

$$
t_{1}^{2} \chi_{\mathrm{QCD}}=c t_{1} M_{\pi}^{2}+b \frac{a^{2}}{t_{1}},
$$

\footnotetext{
${ }^{7} \mathrm{~A}$ recent attempt to extract the topological susceptibility from the local fluctuations of the charge density two-point correlator can be found in Ref. [79].
} 

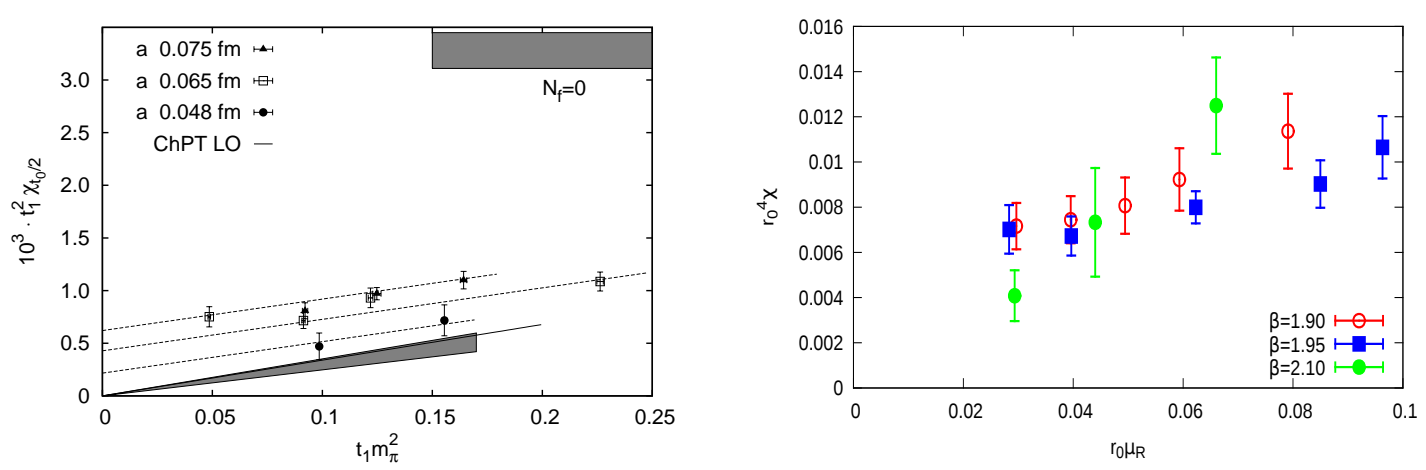

Figure 6: Left: topological susceptibility of the $N_{f}=2$ theory for all ensembles considered in Ref. [68]. Right: the susceptibility for the $N_{f}=2+1+1$ theory for all ensembles simulated in Ref. [80]. Courtesy of Refs. $[68,80]$.

where $t_{1}$ is a reference scale defined analogously to $t_{0}$. The function fits the data well within the (large) statistical errors, see left plot in Figure 6. By taking at face value the result of the fit of $c=2.8(5) \cdot 10^{-3}$ [68], by remembering that at LO in ChPT $c=F^{2} t_{1} / 2 N_{f}$, and by taking the value of $F$ from Eq. (3.3) and $t_{1}=0.061 \mathrm{fm}^{2}$ from Ref. [68], I get $N_{f}=2.06 \pm 0.38$. A result which is in agreement with the expected value, the error being still quite large though.

\section{Conclusions}

Over the last decade there has been an impressive global lattice community effort to reach a precise quantitative understanding of the behaviour of QCD in the chiral regime from first principles. A rather clear and precise picture is emerging.

The spectral density of the Dirac operator in QCD Lite is non-zero at the origin in the continuum and chiral limits. Its value coincide with $M_{\pi}^{2} F_{\pi}^{2} / 2 m$ when $m \rightarrow 0$. This provides a beautiful numerical proof that our picture of spontaneously broken chiral symmetry in QCD is indeed correct. The low modes of the Dirac operator do condense near the origin as dictated by the Banks-Casher mechanism, and their rate of condensation generates the bulk of the pion mass up to quark masses that are about one order of magnitude larger than in Nature.

The topological susceptibility shows the expected suppression with the mass of the light quarks. Within the (so far) large errors, results are compatible with LO ChPT. By now there are many determinations of the QCD low-energy constants obtained by comparing the predictions of ChPT with the precise lattice results with $N_{f}=2, N_{f}=2+1$ and $N_{f}=2+1+1$ flavours.

We have accumulated stronger and stronger evidence that the breaking of the abelian chiral group due to the quantum anomaly is driven by the Witten-Veneziano mechanism. This year it was understood that the recently found definition of the topological charge via the Yang-Mills gradient-flow leads to cumulants of the topological charge distribution which coincide with the universal ones. All numerical results for the topological susceptibility in the SU(3) Yang-Mills theory obtained with the various (proper) definitions agree in the continuum limit within the few percent precision. Its value reproduces the mass of the $\eta^{\prime}$ meson within the expected uncertainty.

On a more theoretical side, the (30 years) long story of defining the topological charge on the lattice comes to a satisfactory end with a simple and elegant solution. Numerical results are 
consistent with this theoretical progress.

Thanks to the conceptual, theoretical and technical advances achieved over the last decade in lattice gauge theory, our femtoscope can explore the chiral regime of QCD with higher and higher precision. This was just a dream only 10-15 years ago, and now is a reality. Hard work is still needed to empower the femtoscope in many other corners of the theory, e.g. the baryon sector.

\section{Acknowledegments}

I wish to thank M. Cè, C. Consonni, G. P. Engel, S. Lottini, M. Lüscher and R. Sommer for an enjoyable collaboration and for many inspiring discussions during the last few years on the topics covered in this talk. I am grateful to my colleagues within the CLS initiative and to those in the Alpha collaboration for generating and sharing the ensembles of gauge configurations with which many of the results reviewed here were obtained. Many thanks to H. Fukaya, K. Jansen, D. J. Murphy and S. Schaefer for sending their results before the conference, and for interesting discussions about their work. Thanks to R. Sommer for a careful reading of this manuscript, and for many suggestions to improve it. It is a pleasure to thank the organizers for their great work in organizing the conference, for being able to create a very stimulating atmosphere, and for giving me the honor to open the conference with this talk.

\section{References}

[1] M. Hasenbusch, Phys. Lett. B 519 (2001) 177 [hep-lat/0107019].

[2] M. Lüscher, Comput. Phys. Commun. 165 (2005) 199 [hep-lat/0409106].

[3] C. Urbach et al., Comput. Phys. Commun. 174 (2006) 87 [hep-lat/0506011].

[4] M. Lüscher, JHEP 0712 (2007) 011 [arXiv:0710.5417 [hep-lat]].

[5] M. Lüscher and S. Schaefer, Comput. Phys. Commun. 184 (2013) 519 [arXiv:1206.2809 [hep-lat]].

[6] M. Lüscher, PoS LAT 2005 (2006) 002 [hep-lat/0509152].

[7] L. Giusti, PoS LAT 2006 (2006) 009 [hep-lat/0702014 [HEP-LAT]].

[8] L. Del Debbio et al., JHEP 0702 (2007) 056 [hep-lat/0610059].

[9] P. Boucaud et al. [ETM Collaboration], Phys. Lett. B 650 (2007) 304 [hep-lat/0701012].

[10] H. Fukaya et al. [JLQCD Collaboration], Phys. Rev. Lett. 98 (2007) 172001 [hep-lat/0702003].

[11] S. Weinberg, Physica A 96 (1979) 327.

[12] J. Gasser and H. Leutwyler, Annals Phys. 158 (1984) 142.

[13] J. Gasser and H. Leutwyler, Nucl. Phys. B 250 (1985) 465.

[14] G. P. Engel et al. [BGR Collaboration], Phys. Rev. D 82 (2010) 034505 [arXiv:1005.1748 [hep-lat]].

[15] P. Fritzsch et al., Nucl. Phys. B 865 (2012) 397 [arXiv:1205.5380 [hep-lat]].

[16] S. Dürr et al. [BMW Collaboration], Phys. Rev. D 90 (2014) 11, 114504 [arXiv:1310.3626 [hep-lat]].

[17] T. Blum et al. [RBC and UKQCD Collaborations], arXiv:1411.7017 [hep-lat].

[18] M. Bruno et al., JHEP 1502 (2015) 043 [arXiv:1411.3982 [hep-lat]]. 
[19] S. Aoki et al., Eur. Phys. J. C 74 (2014) 2890 [arXiv:1310.8555 [hep-lat]].

[20] R. D. Mawhinney and D. J. Murphy, arXiv:1511.04419 [hep-lat].

[21] R. D. Mawhinney and D. J. Murphy, PoS LATTICE2015, 062 (2015).

[22] G. 't Hooft, Phys. Rev. Lett. 37 (1976) 8.

[23] E. Witten, Nucl. Phys. B 156 (1979) 269.

[24] G. Veneziano, Nucl. Phys. B 159 (1979) 213.

[25] E. Vicari and H. Panagopoulos, Phys. Rept. 470 (2009) 93 [arXiv:0803.1593 [hep-th]].

[26] H. Neuberger, Phys. Lett. B 417 (1998) 141 [hep-lat/9707022].

[27] P. Hasenfratz, V. Laliena and F. Niedermayer, Phys. Lett. B 427 (1998) 125 [hep-lat/9801021].

[28] M. Lüscher, Phys. Lett. B 428 (1998) 342 [hep-lat/9802011].

[29] L. Giusti, G. C. Rossi, M. Testa and G. Veneziano, Nucl. Phys. B 628 (2002) 234 [hep-lat/0108009].

[30] L. Giusti, G. C. Rossi and M. Testa, Phys. Lett. B 587 (2004) 157 [hep-lat/0402027].

[31] M. Lüscher, Phys. Lett. B 593 (2004) 296 [hep-th/0404034].

[32] L. Del Debbio, L. Giusti and C. Pica, Phys. Rev. Lett. 94 (2005) 032003 [hep-th/0407052].

[33] T. Banks and A. Casher, Nucl. Phys. B 169 (1980) 103.

[34] H. Leutwyler and A. V. Smilga, Phys. Rev. D 46 (1992) 5607.

[35] E. V. Shuryak and J. J. M. Verbaarschot, Nucl. Phys. A 560 (1993) 306 [hep-th/9212088].

[36] L. Giusti and M. Lüscher, JHEP 0903 (2009) 013 [arXiv:0812.3638 [hep-lat]].

[37] L. Del Debbio et al., JHEP 0602 (2006) 011 [hep-lat/0512021].

[38] K. Cichy, E. Garcia-Ramos and K. Jansen, JHEP 1504 (2015) 048 [arXiv:1412.0456 [hep-lat]].

[39] P. H. Damgaard, K. Splittorff and J. J. M. Verbaarschot, Phys. Rev. Lett. 105 (2010) 162002 [arXiv:1001.2937 [hep-th]].

[40] K. Splittorff and J. J. M. Verbaarschot, Phys. Rev. D 85 (2012) 105008 [arXiv:1201.1361 [hep-lat]].

[41] A. V. Smilga and J. Stern, Phys. Lett. B 318 (1993) 531.

[42] P. H. Damgaard, J. C. Osborn, D. Toublan and J. J. M. Verbaarschot, Nucl. Phys. B 547 (1999) 305 [hep-th/9811212].

[43] P. H. Damgaard and H. Fukaya, JHEP 0901 (2009) 052 [arXiv:0812.2797 [hep-lat]].

[44] S. Necco and A. Shindler, JHEP 1104 (2011) 031 [arXiv:1101.1778 [hep-lat]].

[45] K. Cichy, E. Garcia-Ramos and K. Jansen, JHEP 1310 (2013) 175 [arXiv:1303.1954 [hep-lat]].

[46] G. P. Engel, L. Giusti, S. Lottini and R. Sommer, Phys. Rev. Lett. 114 (2015) 11, 112001 [arXiv:1406.4987 [hep-ph]].

[47] G. P. Engel, L. Giusti, S. Lottini and R. Sommer, Phys. Rev. D 91 (2015) 5, 054505 [arXiv:1411.6386 [hep-lat]].

[48] L. Del Debbio, L. Giusti, M. Luscher, R. Petronzio and N. Tantalo, JHEP 0702 (2007) 082 doi:10.1088/1126-6708/2007/02/082 [hep-lat/0701009]. 
[49] R. Baron et al. [ETM Collaboration], JHEP 1008 (2010) 097 [arXiv:0911.5061 [hep-lat]].

[50] R. Sommer, PoS LATTICE 2013 (2014) 015 [arXiv:1401.3270 [hep-lat]].

[51] M. Della Morte et al. [ALPHA Collaboration], Nucl. Phys. B 713 (2005) 378 [hep-lat/0411025].

[52] S. Capitani et al. [ALPHA Collaboration], Nucl. Phys. B 544 (1999) 669 [hep-lat/9810063].

[53] M. Della Morte et al. [ALPHA Collaboration], Nucl. Phys. B 729 (2005) 117 [hep-lat/0507035].

[54] A. Armoni, M. Shifman and G. Veneziano, Phys. Lett. B 579 (2004) 384 [hep-th/0309013].

[55] A. Armoni, G. Shore and G. Veneziano, Nucl. Phys. B 740 (2006) 23 [hep-ph/0511143].

[56] A. Armoni, M. Shifman, G. Shore and G. Veneziano, Phys. Lett. B 741 (2014) 184 [arXiv:1412.3389 [hep-th]].

[57] E. Seiler, Phys. Lett. B 525 (2002) 355 [hep-th/0111125].

[58] P. Di Vecchia and G. Veneziano, Nucl. Phys. B 171 (1980) 253.

[59] E. Witten, Annals Phys. 128 (1980) 363.

[60] P. Di Vecchia, F. Nicodemi, R. Pettorino and G. Veneziano, Nucl. Phys. B 181 (1981) 318.

[61] R. Kaiser and H. Leutwyler, Eur. Phys. J. C 17 (2000) 623 [hep-ph/0007101].

[62] M. Lüscher, JHEP 1008 (2010) 071 [JHEP 1403 (2014) 092] [arXiv:1006.4518 [hep-lat]].

[63] M. Lüscher and P. Weisz, JHEP 1102 (2011) 051 [arXiv:1101.0963 [hep-th]].

[64] M. Cè, C. Consonni, G. P. Engel and L. Giusti, Phys. Rev. D 92 (2015) 7, 074502 [arXiv:1506.06052 [hep-lat]].

[65] Y. Kikukawa and A. Yamada, Phys. Lett. B 448 (1999) 265 [hep-lat/9806013].

[66] K. Fujikawa, Nucl. Phys. B 546 (1999) 480 [hep-th/9811235].

[67] B. Alles, M. D’Elia and A. Di Giacomo, Nucl. Phys. B 494 (1997) 281 [Nucl. Phys. B 679 (2004) 397] [hep-lat/9605013].

[68] M. Bruno et al. [ALPHA Collaboration], JHEP 1408 (2014) 150 [arXiv:1406.5363 [hep-lat]].

[69] A. Chowdhury, A. Harindranath, J. Maiti and P. Majumdar, JHEP 1402 (2014) 045 [arXiv:1311.6599 [hep-lat]].

[70] K. Cichy et al. [ETM Collaboration], JHEP 1509 (2015) 020 [arXiv:1504.07954 [hep-lat]].

[71] A. Shindler, T. Luu and J. de Vries, arXiv:1507.02343 [hep-lat].

[72] M. Lüscher and F. Palombi, JHEP 1009 (2010) 110 [arXiv:1008.0732 [hep-lat]].

[73] R. Sommer, Nucl. Phys. B 411 (1994) 839 [hep-lat/9310022].

[74] L. Giusti, S. Petrarca and B. Taglienti, Phys. Rev. D 76 (2007) 094510 [arXiv:0705.2352 [hep-th]].

[75] C. G. Callan, Jr., R. F. Dashen and D. J. Gross, Phys. Rev. D 17 (1978) 2717.

[76] L. Del Debbio, H. Panagopoulos and E. Vicari, JHEP 0208 (2002) 044 [hep-th/0204125].

[77] S. Schaefer et al. [ALPHA Collaboration], Nucl. Phys. B 845 (2011) 93 [arXiv:1009.5228 [hep-lat]].

[78] M. Lüscher and S. Schaefer, JHEP 1107 (2011) 036 [arXiv:1105.4749 [hep-lat]].

[79] H. Fukaya et al. [JLQCD Collaboration], PoS LATTICE 2014 (2014) 323 [arXiv:1411.1473 [hep-lat]].

[80] K. Cichy et al. [ETM Collaboration], JHEP 1402 (2014) 119 [arXiv:1312.5161 [hep-lat]]. 